\title{
Iris Localization using Daugman's Intero-Differential Operator
}

\author{
R.B. Dubey \\ ECE, Hindu College \\ of Engg. Sonepat, India
}

\author{
Abhimanyu Madan \\ ECE, Hindu College \\ of Engg. Sonepat, India
}

\begin{abstract}
Iris recognition is regarded as the most reliable and accurate biometric identification system. Most commercial iris recognition systems use patented algorithms developed by Daugman and these algorithms are able to produce perfect recognition rates. These algorithms are based on linear search methods which make the identification process extremely slow and also raise the false acceptance rate beyond the acceptable range. The proposed iris recognition approach consists of an automatic segmentation system that is based on the various algorithms and is able to localise the circular iris and pupil region, occluding eyelids and eyelashes and reflections. Our proposed method has shown out performing results than existing Houghman algorithms.
\end{abstract}

\section{General Terms}

Image Localization, Segmentation, Daughman's InteroDifferential Operator.

\section{Keywords}

Iris Recognition, Daugman's Intero-differential operator, Pupil Boundary, Iris Boundary, Segmentation.

\section{INTRODUCTION}

\subsection{Motivation}

Iris recognition is a special type of biometric system which is used to identify a person by analyzing the patterns in the iris. It is used to recognize human identity through the textural characteristics of one's iris muscular patterns. Although eye color is dependent on heredity but iris is independent even for twins. Out of various biometrics such as finger and hand geometry, face, ear and voice recognition, iris recognition has been proved to be one of the most accurate and reliable biometric modalities because of its high recognition rate [1, 2].

\subsection{The Human Iris}

The iris lies between the cornea and the lens of the human eye. A front view of the iris is shown in Fig. 1 (courtesy from Ref. [3]). The iris is a hole cut close to its centre by a circular aperture called the pupil. The main purpose of the iris is to control the amount of light entering through the pupil via the sphincter and the dilator muscles which in turn adjust the size of the pupil. The iris consists of various layers; the lowest one is the epithelium layer which contains dense pigmentation cells. The stromal layer lies above the epithelium layer and contains blood vessels, pigment cells and the two iris muscles. The externally visible surface of the multi-layered iris has two zones, which are normally of different colors. The collarette divides an outer ciliary zone and an inner pupillary zone in a zigzag form [1, 3, 4].

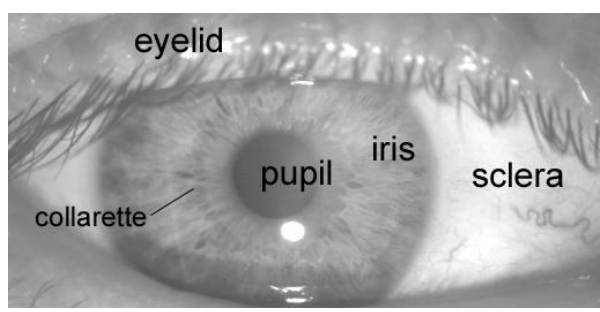

Fig. 1: Front view of the human eye

\subsection{Iris Localization}

The iris localization is achieved by finding the iris boundaries as well as eyelids with location of iris inner and outer boundaries. The iris region shown in Fig. 2 can be approximated by two circles, one for the iris/sclera boundary and another for the iris/pupil boundary. The eyelids and eyelashes normally occlude the upper and lower parts of the iris region $[1,5]$. The success of segmentation depends on the imaging quality of eye images. Noisy and falsely represented data may cause poor recognition rates $[1,5,6,7]$.

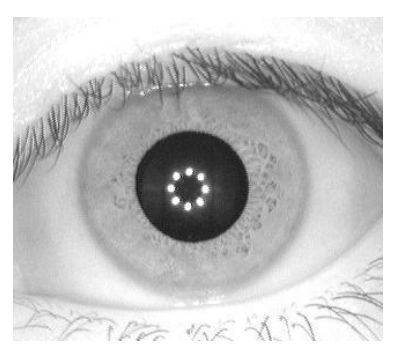

Fig. 2: Human iris

The process of locating and isolating an iris from an image is known as iris localisation or segmentation. The primary task of segmentation is to determine pixels in the image that correspond to the iris region. In Fig. 3 (courtesy from Ref. [29]), the pupillary boundary refers to the boundary separating the pupil from the iris while the limbus boundary refer to the boundary separating the iris from sclera. Normally, segmentation is achieved by detecting the pupillary boundary and the limbus boundary along with the eyelids and eyelashes that can interrupt the contour of the limbus boundary [3]. 


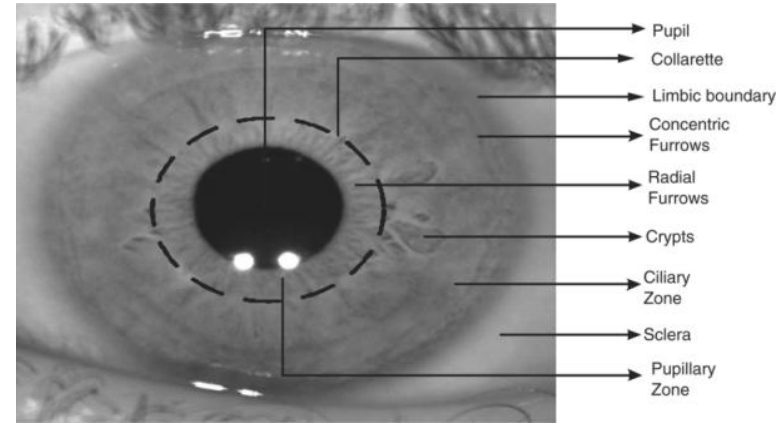

Fig. 3: The anterior surface of the iris

\subsection{Related Work}

Perhaps, the iris recognition is the most reliable biometrics method for person authentication due to several crucial factors including rich and unique textures of the iris, noninvasiveness, stability of iris pattern throughout the person's life time, public acceptance, and availability of user friendly capturing devices [7-10]. Recently, iris localization introduced by various researchers being iris pattern has been proven as a powerful tool to recognize person identification, health status and types of disease [11-15]. From segmented iris features are extracted and analyzed for iris detection. The accuracy of iris recognition heavily depends on the iris segmentation algorithm, which should elicit the effective iris region from an iris image. Localization is a crucial process. Miss localization of inner and outer boundaries of iris causes inaccurate iris segmentation and then failure in further analysis. The most common algorithm used in iris localization is Circular Hough Transform. It has been proven as the best algorithm in localizing iris [16-17, 20]. Q. C. Tian et al. [18] employed modified Hough transform to localize the iris. The modified algorithm has been proven successful to localize iris with short computation time. A. E. Yahya and M. J. Nordin [19] proposed a new technique for iris localization with accuracy of $96.7 \%$. J. Cui et al. [21] proposed an iris localization algorithm based on texture segmentation with achieved accuracy of $99.54 \%$.

\subsection{Contribution}

Iris localization is a process to detect the iris boundary. It is done in two steps. First step is to find the pupillary boundary and the second step is to detect the iris boundary. Original image is converted into binary image. Specular reflection is removed through dilation process, after this maximum area regarding pupil is detected which gives the centre and radius of pupil. A circular contour is drawn using centre and radius of pupil for highlighting the pupillary boundary. Next step is to find the iris boundary using Daughman's Intero- Operator which scans the whole image and detects the iris boundary.

\subsection{Outline of the Paper}

The rest of the paper is organized as follows. In Section 2, various theories for iris localization are described. Section 3 deals with the proposed methodology. The experimental results are discussed in Section 4. Finally, the concluding remarks are given in Section 5.

\section{METHODS USED FOR IRIS LOCALIZATION \\ 2.1 Hough Transform}

The circular Hough transform is used to find the radius; centre coordinates of the pupil and iris regions. Here, parameters $x$ and $y$ are the centre coordinates and $r$ the radius which is used to define any circle by the equation

$x_{c}^{2}+y_{c}^{2}=r^{2}$

A maximum point in the Hough space is equivalent to the radius and centre coordinates of the circle defined by the edge points. The parabolic Hough transform is used to detect the eyelids by approximating the upper and lower eyelids with parabolic arcs, which are represented as;

$\left(-\left(x-h_{j}\right) \sin \theta_{j}+\left(y-k_{j}\right) \cos \theta_{j}\right)^{2}=a_{j}((x-$

$\left.h_{j}\right) \cos \theta_{j}+\left(y-k_{j}\right) \sin \theta_{j}$

For the edge detection step, the derivatives in the horizontal direction for detecting the eyelids and in the vertical direction for detecting the outer circular boundary of the iris are considered [22-25, 30]. Problems had been reported using the Hough transform method. Firstly, it requires threshold values for edge detection and this may result in critical edge points being removed, resulting in failure to detect circles/arcs. Secondly, the Hough transform is computationally intensive due to its brute-force approach and hence not used for real time applications $[22-25,30]$.

\subsection{Active Contour Models}

An active contour works with internal and external forces by deforming moving across an image till equilibrium is established. The contour consisting of various vertices and their positions may be changed by internal and external forces. The internal force depends on the characteristics and an external force is image dependent. The vertex is moved between time $t$ and $t+1$ by the Eqn.

$v_{i}(t+1)=v_{i}(t)+F_{i}(t)+G_{i}(t)$

Here, $F_{i}$ is the internal force; $G_{i}$ is the external force and $v_{i}$ is the position of vertex $I[26,30]$.

In order to locate the pupil region, the internal forces are calibrated to form a contour internally for expanding discrete circle. The external forces are basically used to get the edge information. Eventually, a point interior to the pupil is located from a variance image and then a discrete circular active contour is created with this point as its centre. These forces localized the pupil under the equilibrium condition of internal and external forces $[26,30]$.

\subsection{Eyelash and Noise Detection}

If a resultant point is smaller than a threshold, then this point belongs to an eyelash. Many eyelashes are detected using the variable intensity. Lower the variance value of intensity in a small window than a threshold is an indicator of centre point in an eyelash $[10,23,30]$. 


\section{THE PROPOSED METHODOLOGY}

The proposed methodology uses Daugman's IntegroDifferential Operator (DIO) algorithm [1, 7, 27]. Daugman makes use of an integro-differential operator for locating the circular iris and pupil regions and also the arcs of the upper and lower eyelids. The integro-differential operator is given by Eqn.

$\max _{\left(r, x_{p}, y_{p}\right)}\left|G_{\sigma}(r) * \frac{\partial}{\partial r}\right| \int_{r, x_{0}, y_{0}} \frac{I(x, y)}{2 \pi r} d s$

Here $I(x, y), r, G(r)$ and $s$ are the eye image, radius, Gaussian smoothing function and the contour of the circle respectively. The operator searches for the circular path where there is maximum change in pixel values by varying the radius and centre $x$ and $y$ position of the circular contour to attain precise location of eyelids [27].

Likewise Hough transform, the DIO also uses the first derivatives of the image to find geometric parameters. The DIO uses basic raw derivative information to avoid suffering from any thresholding problems like Hough transform. However, the algorithm fails with the noisy eye images. Iris detection algorithm is divided into two parts: detection of pupil boundary and detection of the iris boundary [27].

\subsection{Detection of the Pupil Boundary}

The process starts for detecting the features of pupil like the centre of the pupil and radius of the pupil. With the help of these features, a contour is drawn which highlights the pupillary boundary. Pupil detection will give a start point for the iris detection. Firstly, the input image is pre-processed to remove the specular reflection from the image. The process is illustrated in Fig. 4.

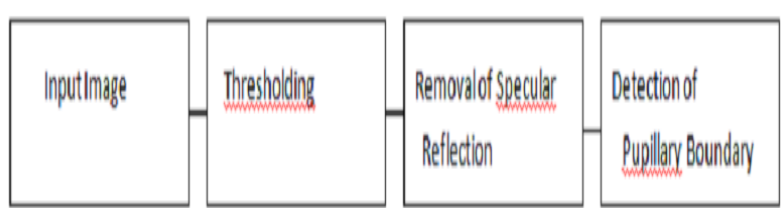

Fig. 4: Pupil detection process

\subsubsection{Thresholding}

It is a very popular method of image segmentation. Here, concept of below thresholding is applied for successful implementation of DIO algorithm. Below thresholding pixels are marked as object pixels which are darker than the background pixels and are called centre pixels. The centre pixels for both the iris and the pupil lie inside the dark pupil region. In some case, the centre pixels might lie in the comparatively lighter region of the iris but not in the white sclera region. A range of $[0,1]$ for intensity values of the pixels in the eye images has been selected, where 0 represents the black pixels and 1 corresponds to white pixels. To achieve better result all the object pixel value below 0.5 are marked before being implementation of the DIO algorithm [28].

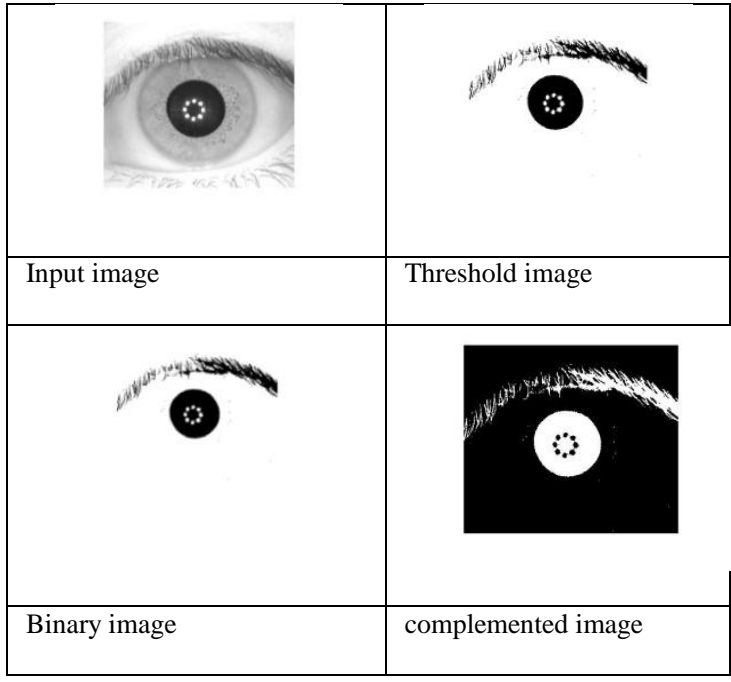

Fig. 5: Various steps involved in pupil detection process

\subsubsection{Removal Specular Reflection}

After thresholding the image or converting the image to binary level, the next step is to remove the specular reflection from the pupil part. In Fig 6, the specular reflection is the white circle in the input image. These are due to reflection of light from the input device through which the image is clicked. For removing this error, a normal morphology processing is used. After this process all these holes are filled and this error is removed.

\subsubsection{Detection of Centroid and Radius of Pupil}

After removing the specular reflection, next step is to find the centre and radius of the pupil. For this, the binary image is converted into a label matrix to find the maximum area. The maximum area regarding the binary image is the filled white pupil circle given in the Fig. 6 . Then the features like centroid and radius of the pupil filled area is obtained. The output of the command is centre coordinate and diameter of the filled pupil circle and a contour is drawn which highlight the boundary of the pupil.

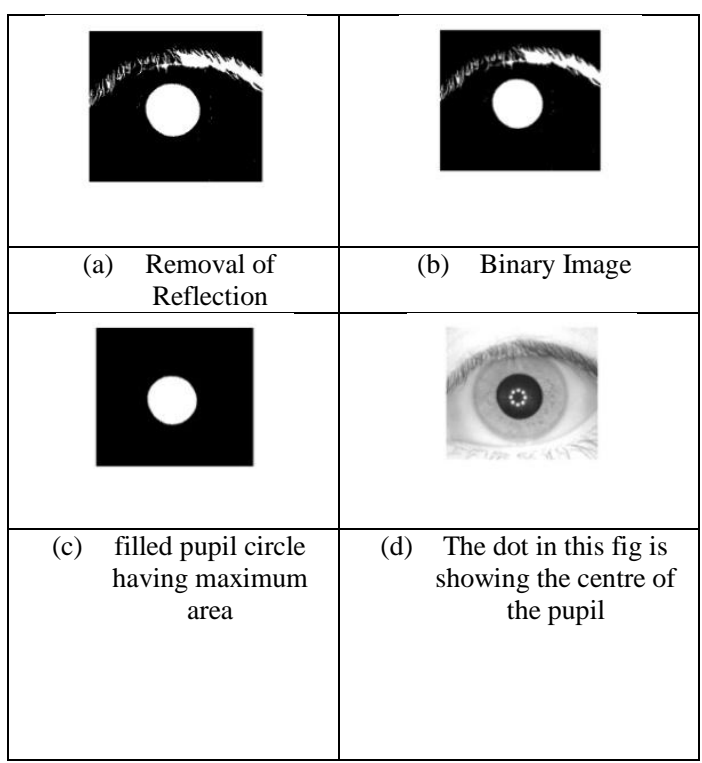




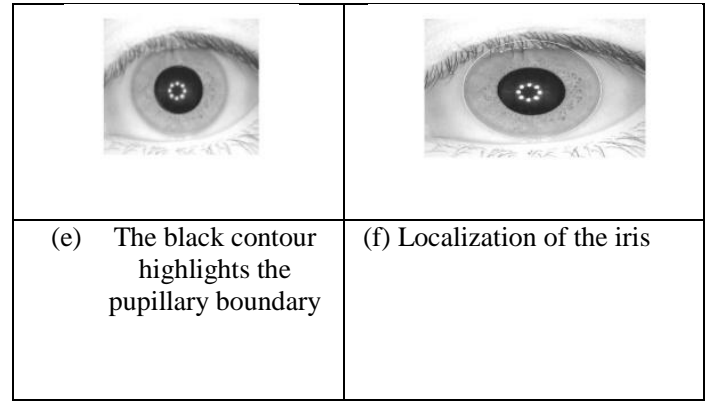

Fig. 6: Various steps involved in iris boundary detection

\subsection{Detection of Iris Boundary}

\subsubsection{The Daugman's Operator}

The operator creates a circular path at maximum change in pixel values by changing the radius $r$ and the centre $(x, y)$ of the circular contour to determine accurate localization [7]. Let the variables $\mathrm{x}, \mathrm{y}$ and $\mathrm{r}$ belong to the ranges $[0 ; \mathrm{X}],[0 ; \mathrm{Y}][0$; $\mathrm{R}$ ] respectively then this method has the computational requirement of the order $[\mathrm{X} \times \mathrm{Y} \times \mathrm{R}]$. Thus, at every pixel, a total of $\mathrm{R}$ scans are necessary to compute the circle parameters using this method. [7, 27].

A pixel by pixel search is carried out over the entire image. At every pixel, the normalized sum of all circumferential pixel values, at increasing radius is determined. At every level of increased radius, the difference between the normalized sums of pixel intensity values at adjacent radii circle is recorded. When the entire search process is completed after summation and differentiation operation, the centre pixel of the iris is found at the change in sum of circumferential pixels intensity values between two highest adjacent contours.

\subsubsection{Detection of Pixels with Minimal Intensity \\ Values}

The threshold image is further scanned, pixel by pixel to determine whether the pixel is a local minimum in that particular pixel's immediate 3-by-3 neighborhood. This means that every pixel intensity is compared to the intensities of the pixels in its immediate nine neighborhood pixels. The pixel with the lowest intensity value amongst these nine pixels is used for further calculations. The rest of the pixels are discarded. The reduction of the number of object pixels, on which the Daugman's operator is applied, makes the iris detection process faster.

\subsubsection{No Circles outside the Image}

The iris border is expected to lie completely inside the input image. During the iris detection process, for every assumed centre pixel the sum of values of circumferential pixels is noted at different levels of radii. Therefore those circular regions of pixels that do not completely lie inside the eye image are neglected. This means that for an assumed centre pixel, the circular contour search is stopped at the radius at which a complete circle of pixels is unable to be formed since the contour pixels coordinates lie outside the coordinates range limited by the image dimensions [7].

\section{RESULTS}

The algorithm is tested with 10 CASIA iris image original database as shown in Fig. 7. The input image is first converted to the binary image which is shown in Fig. 8. This is the first step to find the pupillary boundary. After converting to the binary images, the next step is to convert into their complemented form as shown in Fig. 9 to remove the specular reflections from the images. These specular reflection holes are filled by dilation process which is shown in Fig. 10. After removing the specular reflections the maximum area which gives the pupils are shown in Fig. 11. The maximum area regarding the pupil is used to find the centre of pupil and is shown in Fig. 12. Fig. 13 displays the pupillary boundary by drawing a circle around it. Finally, Fig. 14 shows the iris boundary.

The DIO algorithm is compared with the Hough transform algorithm on the basis of computational time. The computational time for proposed algorithm is $12.056 \mathrm{sec}$. to display the result while for the Hough transform it is about $248.23 \mathrm{sec}$. The algorithm shows far better computational time than the Hough transform algorithm.

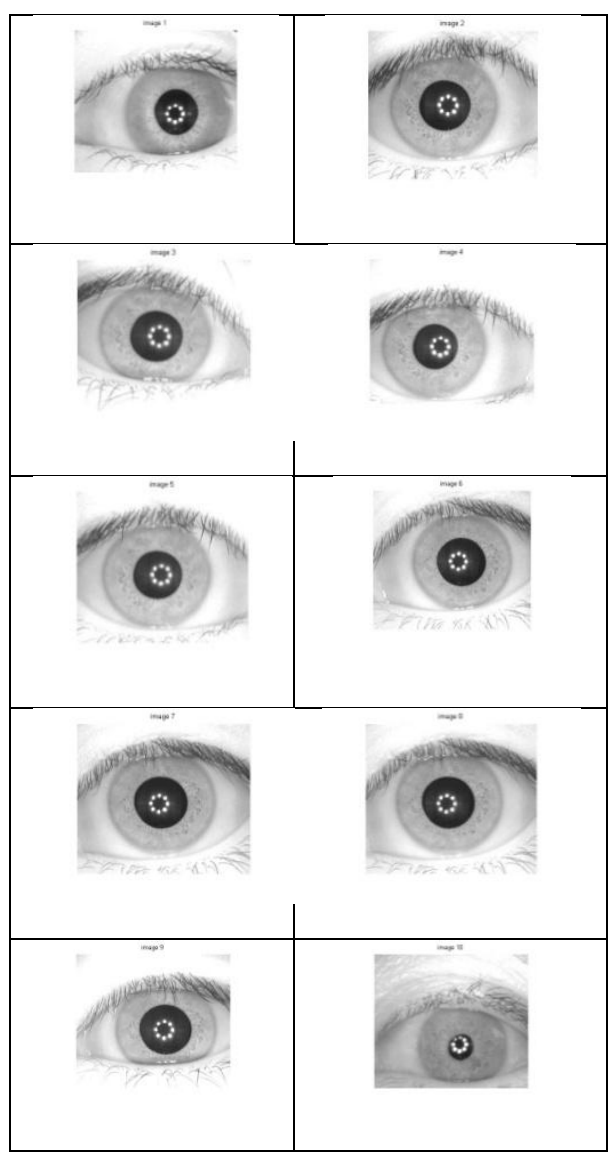

Fig. 7: Original image database 


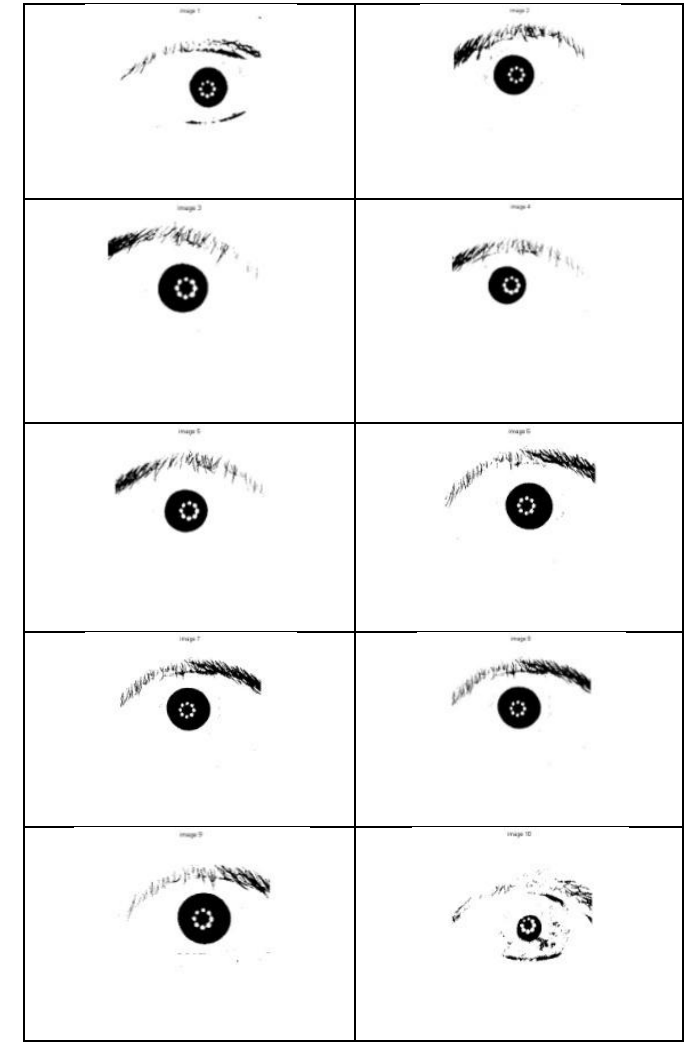

Fig. 8: Binary image

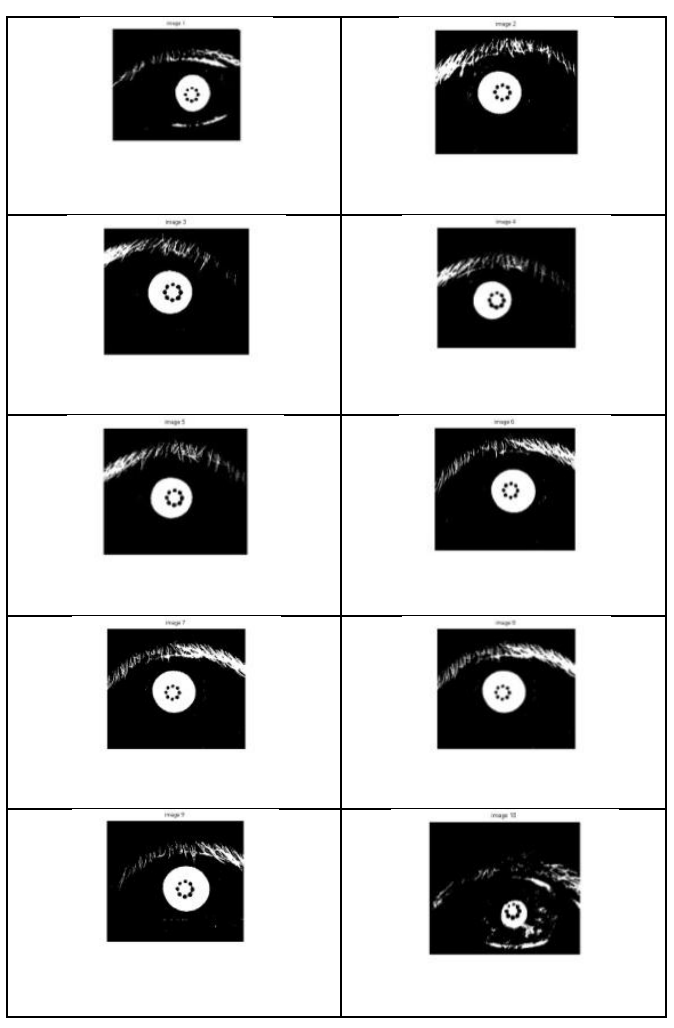

Fig. 9: Complemented image

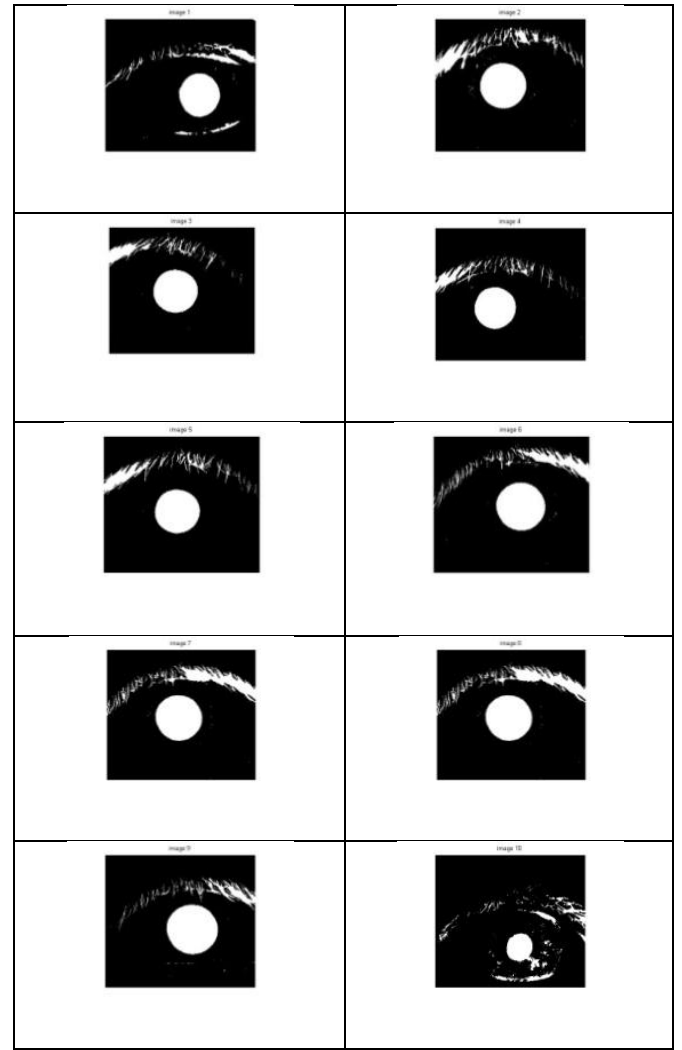

Fig. 10: Filled image

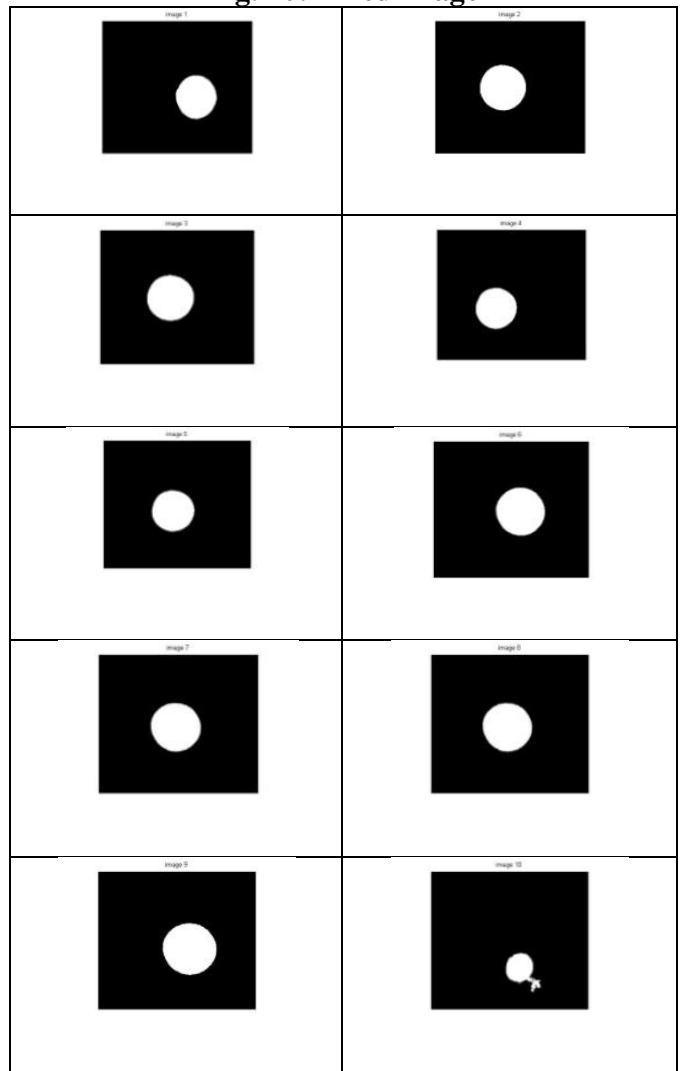

Fig. 11: Maximum area regarding pupil 


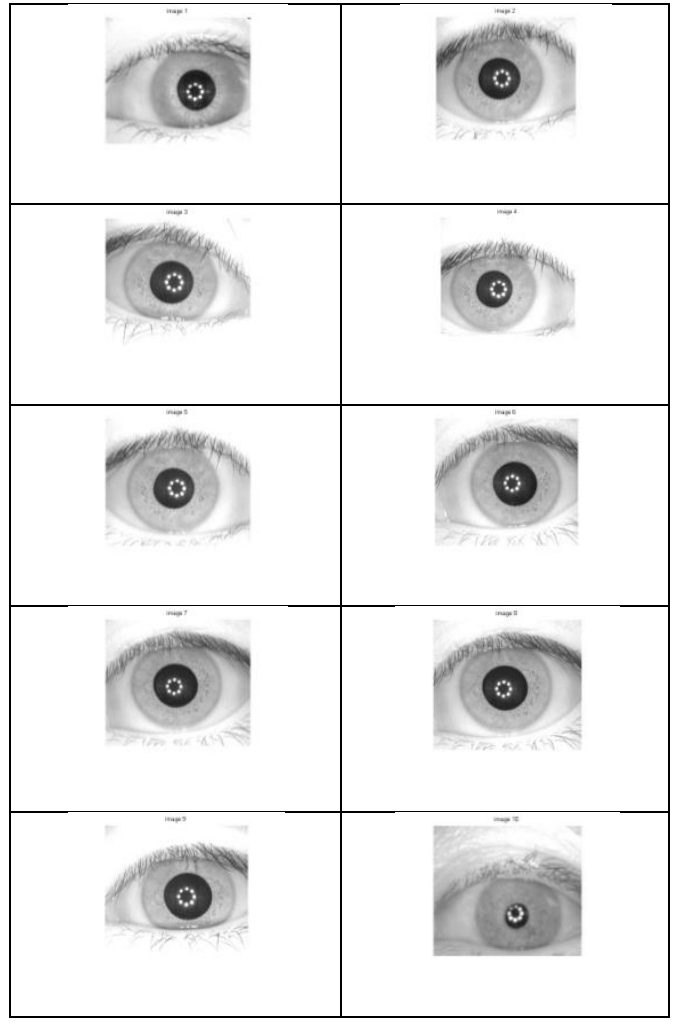

Fig.12: Pupil centre

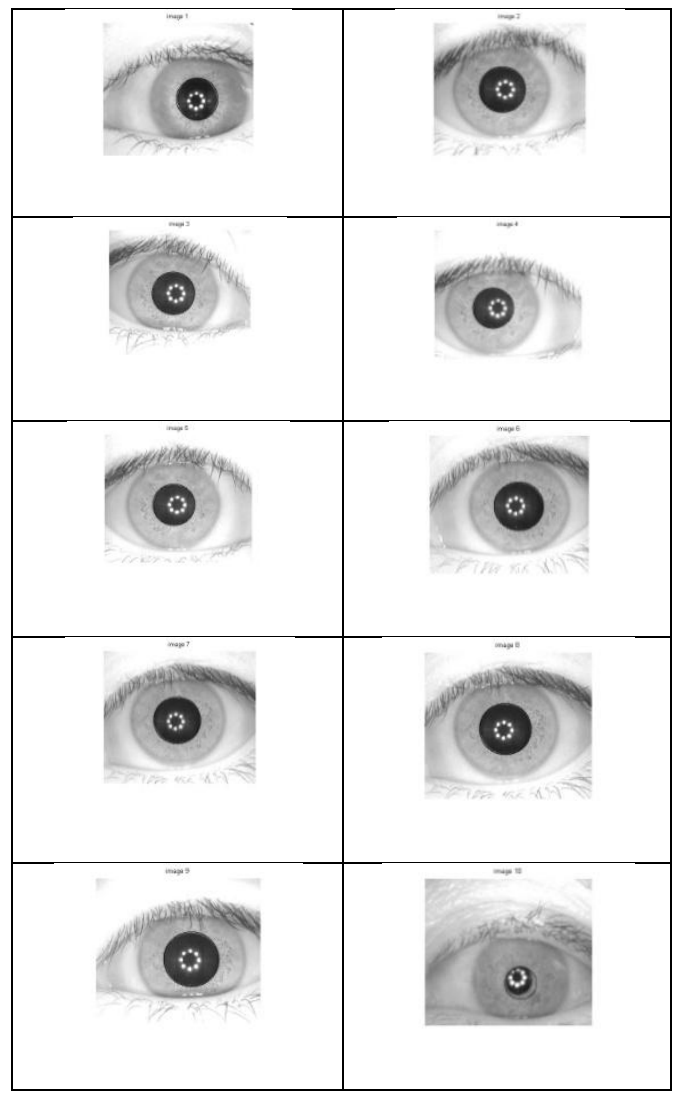

Fig. 13: Contour displaying pupillary boundary

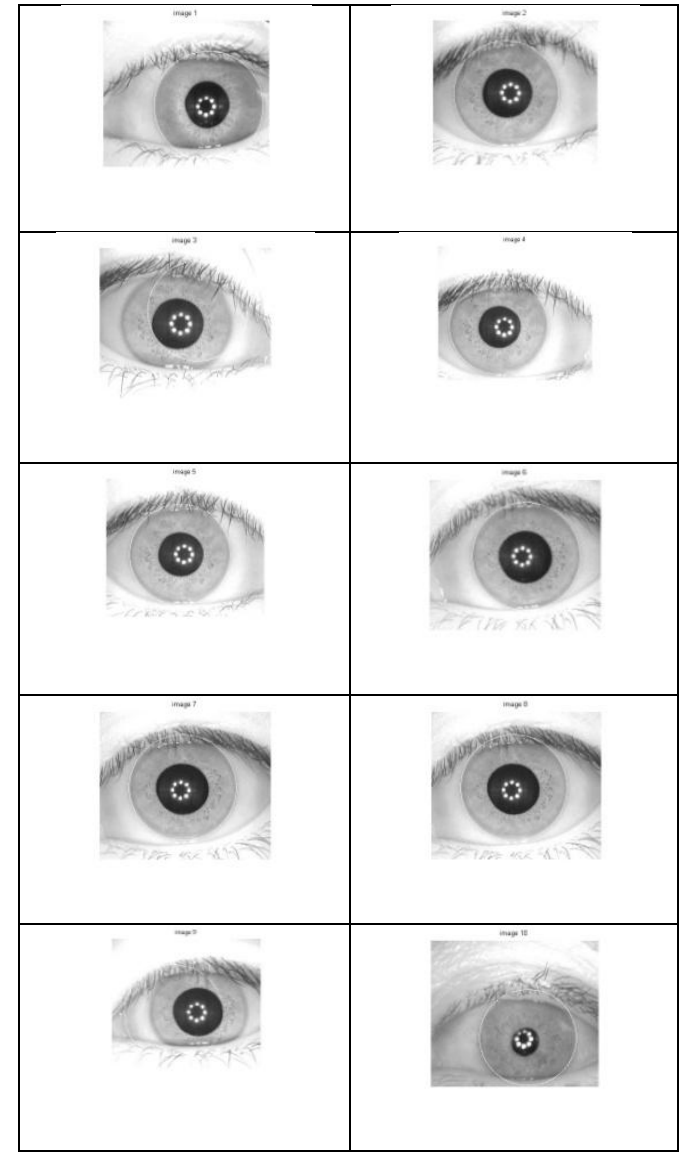

Fig. 14: Contour showing Iris boundary

\section{CONCLUSIONS}

Iris localization is the beginning task in any iris-based biometric authentication system and if the iris part of an eye image is not detected accurately then it leads to an error in overall identification method. This work is focus on efficient and accurate iris localization method for developing better biometric identification system in widespread application areas. The approach addresses the issue of processing iris images where pupil and iris boundaries are not necessarily perfectly circular. To deal with these problems we consider different operations such as, binary image creation, finding all connected component, removal of small connected component, selecting pupil component and finding pupil component for pupil boundary detection and intensity level transformation, dilation and image thresholding. The computational time for proposed algorithm is $12.056 \mathrm{sec}$. while for the Hough transform it is about $248.23 \mathrm{sec}$. The algorithm shows far better computational time than Hough transform algorithm. Our proposed method has shown out performing results than existing algorithms. There are some limitations of this algorithm. Firstly, we have to input the radius range to find the iris boundary. Secondly, the computational time is somewhat high. To overcome these limitations, we are further working on another efficient algorithm for the iris localization. 


\section{REFERENCES}

[1] S. Dey, and D. Samanta, J. Daugman, "How iris recognition works," IEEE Trans on Circuits and Systems for Video Technology, vol. 14, no. 1, pp. 21-30, 2004.

[2] Iris Recognition [Online] Available: http://en.wikipedia.org/wiki/Iris_recognition

[3] E. Wolff. Antomy of the eye and orbit. $7^{\text {th }}$ edition, H. K. Lewis and Ltd.

[4] en.wikipedia.org/wiki/Iris_(anatomy)

[5] S. Dey, and D. Samanta, , "Iris data indexing method using Gabor energy features" IEEE Trans on information forensics and security, vol. 7, no. 4, August 2012.

[6] [6] Casia-IrisV3-Interval Iris Image Database [Online]. Available: http:// www.cbsr.ia.ac.cn/IrisDatabase.htm

[7] J. G. Daugman, "High confidence visual recognition of persons by a test of statistical independence," IEEE Trans. Pattern Anal. Mach. Intell., ol. 15, no. 11, pp. 1148-1161, Nov. 1993.

[8] J. G. Daugman, "How iris recognition works", IEEE Trans Circuits Syst Video Technol, vol. 14, pp. 1-17, 2003

[9] J. G. Daugman, "The importance of being random: statistical principles of iris recognition", Pattern Recognition, vol. 36, pp. 279-91, 2003.

[10] K. Roy, P. Bhattacharya, and C. Y. Suena, "Iris segmentation using variational level set method", Optics and Lasers in Engineering, vol. 49, pp. 578-588, 2011.

[11] A. Harjoko, S. Hartati, and H. Dwiyasa, " A Method for Iris Recognition Based on 1D Coiflet Wavelet", World Academy of Science, Engineering and Technology 56, 2009.

[12] R. T. Al-Zubi and D. I. Abu-Al-Nadi, "Automated personal Identification System Based on Human Iris Analysis", Pattern Analysis Application, vol. 10: pp. 147-164, 2007.

[13] L. Berggren, "Iridology: A critical review", Acta Ophthalmoligica, vol. 63, pp. 1-8, 1985.

[14] R. S. Worrall, "Iridology: Diagnosis or delusion", The Skeptical Inquirer, pp. 23-35, Spring 1983.

[15] R. A. Ramlee and S. Ranjit, "Using Iris Recognition Algorithm, Detecting Cholesterol Presence", 2009 International Conference on Information Management and Engineering.

[16] C. H. Daouk et al., "Iris Recognition", Proceedings of the 2nd IEEE. International. Symposium on Signal Processing and Information Technology, pp. 558-562, 2002.
[17] L. Masek, 2003, Recognition of Human Iris Patterns for Biometric Identification, Thesis School of Computer Science and Software Engineering, University of Western Australia.

[18] Q. C. Tian, Q. Pan, Y. M. Cheng, Q. X. Gao, "Fast Algorithm and Application of Hough Transform in Iris Segmentation", Machine Learning and Cybernetics, 2004. Proceedings of 2004 International Conference on , vol.7, no., pp. 3977- 3980, 26-29 Aug. 2004.

[19] A. E. Yahya, M. J. Nordin, "A New Technique for Iris Localization", International Scientific Conference Computer Science'2008, Information Technology Journal, vol. 7(6):pp. 924-929, 2008.

[20] N. A. Jalil, R. Sahak and A. Saparon, "Iris localization using color segmentation and Circular Hough Transform", IEEE EMBS International Conference on Biomedical Engineering and Sciences pp. 784-788, 2012.

[21] J. Cui, Y. Wang, T. Tan, L. Ma, Z. Sun, "A Fast and Robust Iris Localization Method Based on Texture Segmentation", Proceedings of the 3rd Iberian conference on Pattern Recognition and Image Analysis, Part II, Pages 162 - 169.

[22] R.Wildes, J.Asmuth, G. Green, S. Hsu, R. Kolczynski, J. Matey, S. McBride," A system for automated iris recognition" proceeding IEEE workshop on Apllication of Computer Vision, Sarasota, FL, pp. 121-128, 1994.

[23] W. Kong, D. Zhang, "Accurate iris segmentation based on novel reflection and eyelash detection model", Proc. 2001 Intl Symp Intelligent Multimedia, Video and Speech Proc., 2001.

[24] C. Tisse, L. Martin, L. Torres and M. Robert, "Person identification technique using human iris recognition", Intl Conf. on Vision Interface, 2002.

[25] L. Ma, Y. Wang and T. Tan, "Iris recognition using circular symmetric filters", National Laboratory of Pattern Recognition, Institute of Automation, Chinese Academy of Sciences, 2002.

[26] N. Ritter, "Location of the pupil-iris border in slit-lamp images of the cornea", Proc. Intl Conf. on the Image Analysis and processing, 1999.

[27] J. Daughman," New Methods in iris recognition," Cybernetics, vol. 37, no. 6, October 2007.

[28] R. C. Gonzalez and R.E. Woods, Digital Image Processing, $2^{\text {nd }}$ ed. Englewood Cliffs, NJ: Prentice-Hall, 2002.

[29] Samir Shah and Arun Ross,, "Iris segmentation using geodesic active contours", IEEE Trans on Information Forensics and Security, vol. 4, no. 4, December 2009.

[30] http://people.csse.uwa.edu.au/pk/studentprojects/libor/Li borMasekThesis.pdf 